\title{
Baroclinic toroidal quasi-geostrophic vortices
}

\author{
Jean N. Reinaud ${ }^{a)}$ \\ University of St Andrews, Mathematical Institute, North Haugh, \\ St Andrews KY169SS, UK
}

We investigate the stability and nonlinear evolution of two tori of opposite-signed uniform potential vorticity, located one above the other, in a three-dimensional, continuously-stratified, quasi-geostrophic flow. We focus on the formation of hetons as a result of the destabilisation of the tori of potential vorticity. Hetons are pairs of vortices of opposite-sign lying at different depths capable of transporting heat, momentum and mass over large distances. Particular attention is paid to the condition under which the hetons move away from their region of formation. We show that their formation and evolution depend on the aspect ratio of the tori, as well as the vertical gap separating them. The aspect ratio of a torus is the ratio of his major (centreline) radius to its minor (cross-sectional) radius. Pairs of thin opposite-signed potential vorticity tori self-organise into a large number of hetons. On the other hand, increasing the vertical gap between the tori decreases the coupling between the opposite-signed vortices forming the hetons. This results in a more convoluted dynamics where the vortices remain near the centre of the domain.

Keywords: Quasi-Geostrophy, hetons, vortex interactions

${ }^{a)}$ Electronic mail: jean.reinaud@st-andrews.ac.uk 


\section{INTRODUCTION}

Large scale oceanic and atmospheric flows are strongly influenced by the background planetary rotation and by the stable density stratification of the fluid. When these effects are dominant, the flow remains in near geostrophic and hydrostatic balance. In this context, the flow is controlled by a single scalar quantity, the potential vorticity which is materially conserved in absence of viscous and diabatic effects. Vortices can be seen as masses of potential vorticity anomaly (hereinafter referred to as PV for simplicity). In the oceans, vortices interact with external flows, bathymetry and coasts. They also often interact with other vortices. A vortex sometimes couples with another vortex and the vortices move as a pair. When the vortices have opposite sign, they form dipoles which can self-propagate in the flow. The evolution of opposite-signed baroclinic pairs of discrete vortices has first been studied in a two-layer flow ${ }^{1}$ as well as in a continuously stratified fluid. ${ }^{2}$ Such baroclinic dipoles are known as hetons, and their self-propagation provides a mechanism to transport heat, as well as momentum or tracers, in the oceans over large distances. ${ }^{3}$ We differentiate straight hetons and tilted hetons. Straight hetons have their vortices aligned in the vertical direction. The vortices of tilted hetons are horizontally offset. In the latter situation, the horizontal offset creates a dipolar moment which makes the hetons self-propagate.

Finite core hetons are sensitive to baroclinic instability. The linear stability of straight hetons has been addressed in a two layer flow ${ }^{4,5}$ as well as in a continuous stratification. ${ }^{6}$ The introduction of a vertical offset between the vortices of the hetons has a stabilising effect. ${ }^{6}$ The same is true if the hetons are tilted as the increase of the horizontal offset between the vortices of the hetons decreases the strength of their interaction. ${ }^{7}$ The stability of tilted hetons in a two-layer flow has also been analysed ${ }^{8}$ and their nonlinear evolution investigated. ${ }^{9}$ Additionally, hetons have shown to be stable on the $\beta$-plane over a vast range of parameters. ${ }^{10}$ The interaction of discrete hetons has been the topic of several studies. ${ }^{3,11}$ The stability of pairs of discrete hetons known as hetonic quartets has been addressed in the work. ${ }^{12}$ Hetons can interact with other hetons and form complex secondary structures as it has been shown in a two-layer flow, ${ }^{13}$ and in a continuously stratified flow. ${ }^{14,15}$

Hetons are known to form from the presence of patches of either fresh water or brine under ice in the oceans ${ }^{16}$ or from convective effects. ${ }^{17}$ They can also form from baroclinic instability of a coastal current, ${ }^{18,19}$ or from unstable jets. ${ }^{20,21}$ Hetons have been observed to form from 
the coupling of Gulf Stream rings and deep anticyclones, ${ }^{22}$ and from the Mediterranean Water undercurrents in the Gulf of Cadiz. ${ }^{23,24}$

In this paper, we focus on the formation of hetons from the destabilisation of tori of uniform PV. Tori of PV induce shear zones. The initial distribution of PV is axisymmetric and therefore corresponds to a steady (albeit unstable) state. Tori of PV are sometimes observed in planetary atmospheres. A prime example is the annular polar vortex on Mars ${ }^{25-27}$ which may persist due to thermal relaxation. In absence of such a forcing, annular vortices, or tori of PV, are unstable and self-organise into arrays of vortices. ${ }^{28-30}$ This mechanism has been put forward as a possible explanation of the presence of regular arrays of cyclonic vortices observed ${ }^{31}$ over the poles of Jupiter. ${ }^{32}$ We generalise here the study to pair of baroclinic tori of PV. If two tori of opposite-signed PV are located at two different depths, their destabilisation and their subsequent self-organisation into vortex arrays may lead to the formation of hetons.

The evolution of the baroclinic tori of PV shows some similarity with the one of nested baroclinic annular vortices. Such vortices were studied in a two-layer flow as a generalisation of the barotropic nested vortices. ${ }^{33}$ Nested vortices are models of vortex rings found in the oceans. Related works also include the study of annular vortices around an island. ${ }^{34,35}$

There is also a practical advantage in focusing on initial conditions consisting of tori of PV: they are steady states where the PV has compact support (there is a finite volume of PV). The flow can therefore be studied using Lagrangian methods in an explicitly unbounded domain. This means that the hetons can move without interacting with periodic images or other boundary conditions at finite distance which would otherwise have to be imposed in an Eulerian computational box. Two infinite rectilinear filaments of PV of opposite sign also induce an unstable baroclinic shear flow. ${ }^{36}$ But in this case, the periodic conditions affect the long-term evolution of the flow, and only the linear stability and the early stages of the nonlinear evolution can be effectively studied.

In the present work, we show that the pair of tori is sensitive to mixed barotropic and baroclinic unstable modes, due to the presence of both horizontal and vertical shear in the flow, and notably the change of sign of PV in the vertical direction. The azimuthal wavenumber of the most amplified mode first depends on the aspect ratio of the tori, with thin tori amplifying instabilities with higher wavenumbers. It also depends on the vertical distance separating the two tori. Increasing the vertical gap separating the two tori decreases 
the vertical shear they induce on each other.

The paper is organised as follows. The mathematical and numerical models are introduced in Sec. II. The linear stability analysis of the flow is presented in Sec. III. Results from nonlinear simulations of the unstable tori are presented in Sec. IV. Conclusions are given in Sec. V.

\section{STUDY SET-UP}

\section{A. Mathematical model}

We denote $\mathrm{Fr}=U /(N H)$ the Froude number and $R o=U /(f L)$ the Rossby number. Here $U$ is a typical horizontal velocity scale, $H$ and $L$ are typical horizontal and vertical length scales, $N$ is the buoyancy frequency and $f$ is the Coriolis frequency. We assume both $f$ and $N$ constant for simplicity. If $F r^{2} \ll R o \ll 1$, Euler's equations for an incompressible, rapidly-rotating, stably-stratified fluid under the Boussinesq approximation can be asymptotically expanded in terms of $R o$. Keeping terms up to $\mathcal{O}\left(R_{0}\right)$ we obtain the quasi-geostrophic (QG) model,

$$
u=-\frac{\partial \varphi}{\partial y}, \quad v=\frac{\partial \varphi}{\partial x}
$$

where the streamfunction $\varphi$ relates to the PV $q$,

$$
q=\frac{\partial^{2} \varphi}{\partial x^{2}}+\frac{\partial^{2} \varphi}{\partial y^{2}}+\frac{\partial^{2} \varphi}{\partial z^{2}}
$$

In equation (2), $z=z_{p} N / f$ is a stretched vertical coordinate, where $z_{p}$ is the physical vertical coordinate. Finally, PV is materially conserved for an adiabatic and inviscid flow,

$$
\frac{\mathrm{D} q}{\mathrm{D} t}=0
$$

where $\mathrm{D} / \mathrm{D} t=\partial / \partial t+\boldsymbol{u} \cdot \boldsymbol{\nabla}$ is the material derivative. ${ }^{37}$

It should be noted that while the vertical velocity $w$ is not zero in the QG model, it is too small to contribute to the advection of $\mathrm{PV}$.

Equation (2) can formally be inverted

$$
\varphi(\boldsymbol{x})=\iiint_{V} \frac{q\left(\boldsymbol{x}^{\prime}\right)}{\left|\boldsymbol{x}-\boldsymbol{x}^{\prime}\right|} \mathrm{d}^{3} \boldsymbol{x}^{\prime},
$$


leading to

$$
\boldsymbol{u}(\boldsymbol{x})=-\frac{1}{4 \pi}\left(\frac{\partial}{\partial y},-\frac{\partial}{\partial x}\right) \iiint_{V} \frac{q\left(\boldsymbol{x}^{\prime}\right)}{\left|\boldsymbol{x}-\boldsymbol{x}^{\prime}\right|} \mathrm{d}^{3} \boldsymbol{x}^{\prime}
$$

\section{B. Numerical set-up}

In practice, the fluid domain is discretised in the vertical direction by a finite, yet typically large, number of horizontal layers. In each layer, and for each region of uniform PV, the integration in the vertical direction in equation (5) is done explicitly over the thickness of each layer. Then the remaining (horizontal) surface integrals are converted into line integrals along the contours bounding the uniform PV. The technique, originated for two-dimensional flows, ${ }^{38}$ is the QG contour dynamics method. ${ }^{39}$

The linear stability of the flow is investigated by analysing the deformation modes of the vortex bounding horizontal contours. The deformation of the contours is taken in the form

$$
(\tilde{x}, \tilde{y})=\left(x_{e}, y_{e}\right)+\tilde{\gamma}_{m} \mathrm{e}^{\sigma t+\mathrm{i} m \theta} \frac{\left(-\mathrm{d} y_{e} / \mathrm{d} \theta, \mathrm{d} x_{e} / \mathrm{d} \theta\right)}{\left(\mathrm{d} x_{e} / \mathrm{d} \theta\right)^{2}+\left(\mathrm{d} y_{e} / \mathrm{d} \theta\right)^{2}}
$$

where $(\tilde{x}, \tilde{y})$ are the perturbed horizontal coordinates along the contours, $\left(x_{e}, y_{e}\right)$ are the coordinates at equilibrium. $\theta$ is the azimuthal angle, $\tilde{\gamma}_{m}$ is the perturbation complex amplitude and has the dimension of an area, $m$ is the azimuthal wavenumber of the mode considered, and $\sigma=\sigma_{r}+\mathrm{i} \sigma_{i} \in \mathbb{C}$ is the complex frequency of the mode. Its real part $\sigma_{r}$ is the growth rate and its imaginary part $\sigma_{i}$ is the (real) frequency. For each value of $m$, the linearised system of equations for the deformation of the contours gives rise to a $2 n_{c}$-real eigenvalue problem, where $n_{c}$ is the number of contours used to discretise the PV distribution. ${ }^{32}$ The eigenvalues are the complex frequencies $\sigma$, while the eigenvectors are the perturbation com-

plex amplitudes $\tilde{\gamma}_{m}$ and give the spatial structure of the modes. The mode $m=1$ represents a displacement of the contours.

For the nonlinear simulation of the flow evolution, the vortex bounding contours are followed in a Lagrangian way. Time integration is performed using the standard fourthorder Runge-Kutta scheme with a time step $\Delta t=\pi /\left(20 q_{\max }\right)$, where $q_{\max }$ is the maximum (in absolute value) PV The complexity of the vortex bounding contours is kept under control by a topological procedure called 'Contour Surgery'. ${ }^{28}$ 


\section{General geometry}

We consider two, geometrically identical, tori of uniform PV. Both tori are axisymmetric with respect to the vertical axis. The tori have the same major (centreline) radius $R_{0}$ and the same minor (cross-sectional) radius $r_{0}$. The two tori are separated in the vertical direction by a gap $\Delta z$, with no $\mathrm{PV}$. We define $\ell_{z}$ the vertical gap normalised by the tori cross-sectional diameter $\ell_{z}=\Delta z /\left(2 r_{0}\right)$. The tori have opposite-signed uniform $\mathrm{PV} q= \pm 2 \pi$. The time scale $T$ is set by the PV, $T=2 \pi / q$. It should be noted that a sphere of uniform $\mathrm{PV} q$ has a turnover period of $T_{\text {over }}=6 \pi / q$, while an infinite vertical cylinder has a turnover period $T_{\text {over }}=4 \pi / q$. The length scale of the problem is the full height of the pair of tori $H=4 r_{0}+\Delta z$. There is no dynamical asymmetry between a cyclonic and an anticyclonic vortex in the QG model, apart from the direction of rotation. The asymmetry in behaviour between cyclones and anticyclones only appears when ageostrophic effects are taken into account. This is beyond the QG model, and beyond the scope of the present study. For constant buoyancy frequency $N$, and under the Boussinesq approximation, the system also remains symmetric in the vertical direction. Hence, whether the torus with positive PV is on the top or on the bottom is dynamically irrelevant. The general geometry of the configuration is summarised in Fig. 1.

\section{LINEAR STABILITY}

This section is devoted to the linear stability analysis of the pair of tori. Each torus is mapped by 51 layers in the vertical direction. This vertical resolution is the same as the one used for the nonlinear simulations. Tests were performed at higher resolution (mapping each torus by 85 layers) and results show that 51 layers are enough to accurately capture the stability properties of the flow. In each layer, the vortex boundary consists of two contours: one for its inner boundary and one for its outer boundary. There are therefore 204 contours discretising the pair of tori. Each contour is discretised using $n_{p}=400$ nodes (600 nodes were used for the 85-layer tests). The vertical gap $\Delta z$ is set, for convenience, to an integer multiple of the layer thickness, although this choice is not imposed by the method. The normalised values for the vertical gap considered are $\ell_{z}=0$ (the two tori touch), $\ell_{z}=13 / 51 \simeq 0.25, \ell_{z}=25 / 51 \simeq 0.5$ and $\ell_{z}=1$. We analyse the linear stability 

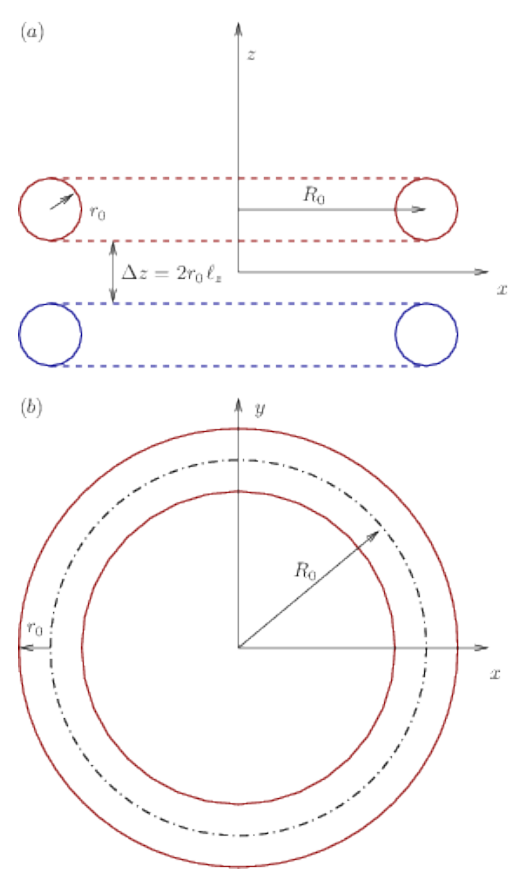

FIG. 1. General geometry of the configuration and definition of the geometrical parameters: $(a)$ side view, (b) top view.

of tori in the range of aspect ratio $1.1 \leq R_{0} / r_{0} \leq 12$. The growth rates of the ten most amplified modes for each value of the tori aspect ratio $R_{0} / r_{0}$ are presented in Fig. 2 .

Results show that all configurations are sensitive to multiple unstable modes. In all cases investigated, modes appear as pairs of repeated eigenvalues, i.e. the complex eigenvalues $\sigma$ are double roots to the eigenvalue problem. We associate such pairs of equal eigenvalues to one single mode. It should also be noted also that if $\sigma=\sigma_{r}+\mathrm{i} \sigma_{i}$ is a complex eigenvalue, so is its complex conjugate $\sigma^{*}=\sigma_{r}-\mathrm{i} \sigma_{i}$. Finally all unstable modes $\sigma$ with $\sigma_{r}>0$ have an associated stable counterpart, $\sigma^{\prime}=-\sigma$, with $\sigma_{r}^{\prime}=-\sigma_{r}<0$.

As one expects, the maximum growth rate of instability typically decreases as the relative vertical gap $\ell_{z}$ is increased. This is due to the decrease of the vertical shear that the tori induce onto each other. This stresses the destabilising effect of the vertical shear, through baroclinic instability. It should be noted that this is similar to the effects of an increase of the horizontal separation between two vortices forming a barotropic vortex dipole ${ }^{40,41}$. This pattern was also observed in two-layer finite-core hetons ${ }^{8}$, for continuously stratified tilted hetons $^{7}$, as well as in two- and three-layer modons ${ }^{10}$ and in point-vortex hetonic quartets ${ }^{12}$.

We also note that except when $R_{0} / r_{0}$ is small, the amplitude of the growth rate for 

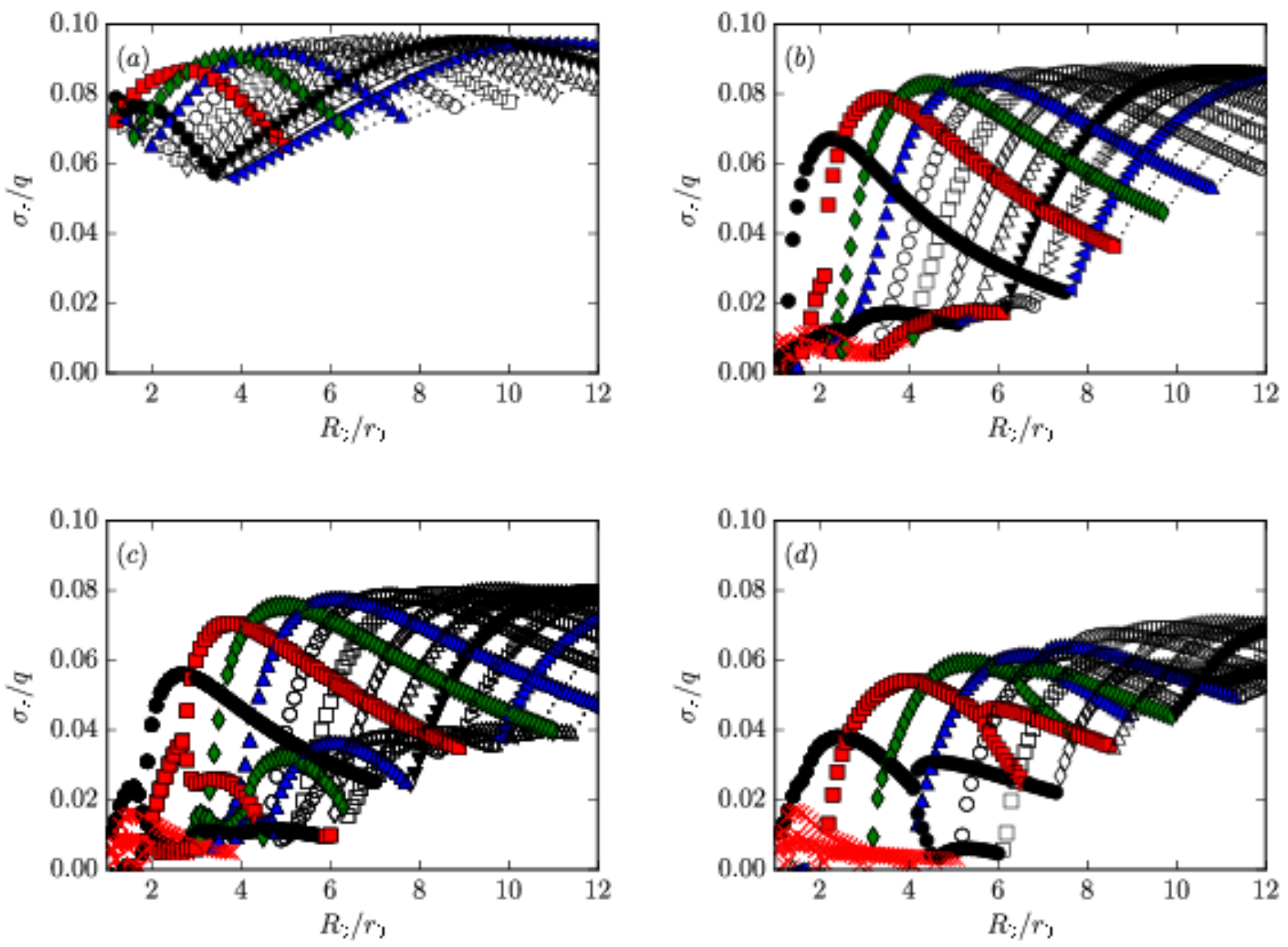

FIG. 2. Normalised growth rates $\sigma_{r} / q$ for the ten most amplified modes for each value of the tori aspect ratio $R_{0} / r_{0}$, for $\ell_{z}=0:(a), 13 / 51:(b), 25 / 51:(c), 1:(d)$. The azimuthal wavenumber of the modes are $m=1: \times, 2: \bullet, 3: \boldsymbol{\square}, 4: \diamond, 5: \boldsymbol{\Delta}, 6: \circ, 7: \square, 8: \diamond, 9: \triangle, 10: \nabla, 11: \triangleright, 12: \triangleleft$. All higher modes are indicated by dotted lines.

the most amplified mode varies little with $R_{0} / r_{0}$, reaching a plateau of $\sigma_{r}^{\max } \sim 0.093$ (for $\left.\ell_{z}=0\right), 0.083\left(\ell_{z}=13 / 25\right), 0.08\left(\ell_{z}=25 / 51\right), 0.07\left(\ell_{z}=1\right)$, at least for the range of tori aspect ratio considered. This value is therefore set by the available horizontal and vertical shears present in the flow which mostly depend on $q$ and $\ell_{z}$. For $\ell_{z}=1$, the order of magnitude of the growth rates are comparable to the one computed for a single torus, ${ }^{32}$ indicating the weakening of the vertical shear induced by the tori. The growth rates differ in their details though.

On the other hand, the wavenumber of the most amplified unstable modes varies with $R_{0} / r_{0}$. Unstable modes with increasing wavenumbers $m$ appear as $R_{0} / r_{0}$ is increased, as ob- 
served for a single torus of PV. ${ }^{32}$ Indeed, as the mean perimeter $2 \pi R_{0}$ of the tori is increased, a larger number of characteristic wavelengths $\lambda$ for the perturbations (proportional to the cross-sectional radius $r_{0}$ ) fit along the torus. This is true for all values of $\ell_{z}$ investigated. For each $m$ (except $m=1,2)$, there is a critical threshold $\left(R_{0} / r_{0}\right)_{m}^{c}$ for the tori aspect ratio below which the mode is neutrally stable, with $\sigma_{r}(m)=0 . \sigma_{r}(m)>0$ for $R_{0} / r_{0}>\left(R_{0} / r_{0}\right)_{m}^{c}$. For $\ell_{z}=0$ the pair of tori is sensitive to unstable modes $m=3,4$ even if $R_{0} / r_{0}$ is small and the threshold would corresponds to $R_{0} / R_{0}<1$, where the vortices are no longer tori. We can see that the value of this threshold increases as $\ell_{z}$ is increased. This is characteristic of the influence of the vertical shear, and this shift is also observed for compact hetons. ${ }^{6}$

For $R_{0} / r_{0} \gtrsim 6$ (typically), several unstable modes with different wavenumbers have growth rates of roughly the same magnitude. This indicates that the destabilisation of the tori of $\mathrm{PV}$ is not dominated by the growth of single monochromatic modes. There are also numerous secondary modes with much smaller growth rates. These modes have arguably little change to fully develop before the growth of the dominant modes fundamentally modifies the flow. For $\ell_{z}=1$, some of the branches of secondary modes bifurcate into two distinct branches. This is the case, for example, for the branch for $m=2$ and $R_{0} / r_{0} \geq 4.1$, $m=3$ and $R_{0} / r_{0} \geq 5.7, m=4$ and $R_{0} / r_{0} \geq 6.5$. The bifurcations occur when the frequency $\sigma_{i}$ of a couple of complex conjugate modes vanishes. Similar bifurcations are observed for hetonic rectilinear filaments of $\mathrm{PV} .{ }^{36}$

\section{NONLINEAR EVOLUTION}

We next turn our attention to the nonlinear evolution of the unstable pairs of tori. As mentioned in Section II, the computational domain is explicitly infinite in all three dimensions of space, and the boundary condition is set by the fact that velocity goes to 0 as $r=\sqrt{x^{2}+y^{2}+z^{2}} \rightarrow \infty$. Due to the fact that the tori are typically sensitive to multiple unstable modes with different azimuthal wavenumbers $m$, we do not impose at $t=0$ a monochromatic perturbation on the vortex bounding contours. We instead let perturbations grow from the low amplitude numerical noise. 
TABLE I. Normalised growth rates $\sigma_{r} / q$ and corresponding azimuthal wavenumber $m$ for the two most amplified modes for thick tori, $R_{0} / r_{0}=3$.

\begin{tabular}{lcr}
\hline \hline$\ell_{z}$ & $\sigma_{r} / q \times 10^{-2}$ & $m$ \\
\hline 0 & 8.8 & 4 \\
0 & 8.6 & 3 \\
$13 / 51$ & 7.7 & 3 \\
$13 / 51$ & 6.1 & 2 \\
$25 / 51$ & 6.0 & 3 \\
$25 / 51$ & 5.5 & 2 \\
1 & 4.6 & 3 \\
1 & 3.6 & 2 \\
\hline \hline
\end{tabular}

\section{A. Thick tori, $R_{0} / r_{0}<7.2$}

We start by presenting the fate of thick tori of aspect ratio $R_{0} / r_{0}=3$. The evolution of the contours bounding the PV tori is shown in Fig. 3 for $\ell_{z}=0,13 / 51,25 / 51$, and 1 .

As shown by the linear stability analysis, the instability threshold $\left(R_{0} / r_{0}\right)_{m}^{c}$ for a mode with a given wavenumber $m$ increases as $\ell_{z}$ is increased. Hence, for a given aspect ratio $R_{0} / r_{0}$ tori vertically separated by a small gap $\ell_{z}$ are more likely be sensitive to high wavenumber modes. The normalised growth rates $\sigma_{r} / q$ and corresponding azimuthal wavenumbers $m$ for the two most amplified modes for $R_{0} / r_{0}=3$ are given in table I. Fig. 3 shows the initial growth of the dominant mode $m=4$ for $\ell_{z}=0$, and $m=3$ for $\ell_{z}=13 / 51$. For $\ell_{z}=25 / 51$, the growth rates of both modes $m=2$ and $m=3$ are similar (see table I), and both modes grow simultaneously. In all cases but $\ell_{z}=1$, the tori self-organise into elongated or compact vortices near the centre of the domain. The configuration eventually splits into two hetons which move away from the centre. This late evolution is similar to the one of compact hetons. ${ }^{6}$

We next identify the coherent vortices present in the flow for all times $t$. Vortices are defined as contiguous regions of PV. We then determine the volume of each vortex as well as its geometrical centre. The evolution of the vortex volumes and the trajectory of their centres are presented in Fig. 4 for $R_{0} / r_{0}=3$ with $\ell_{z}=13 / 51$ and with $25 / 51$. Panels 

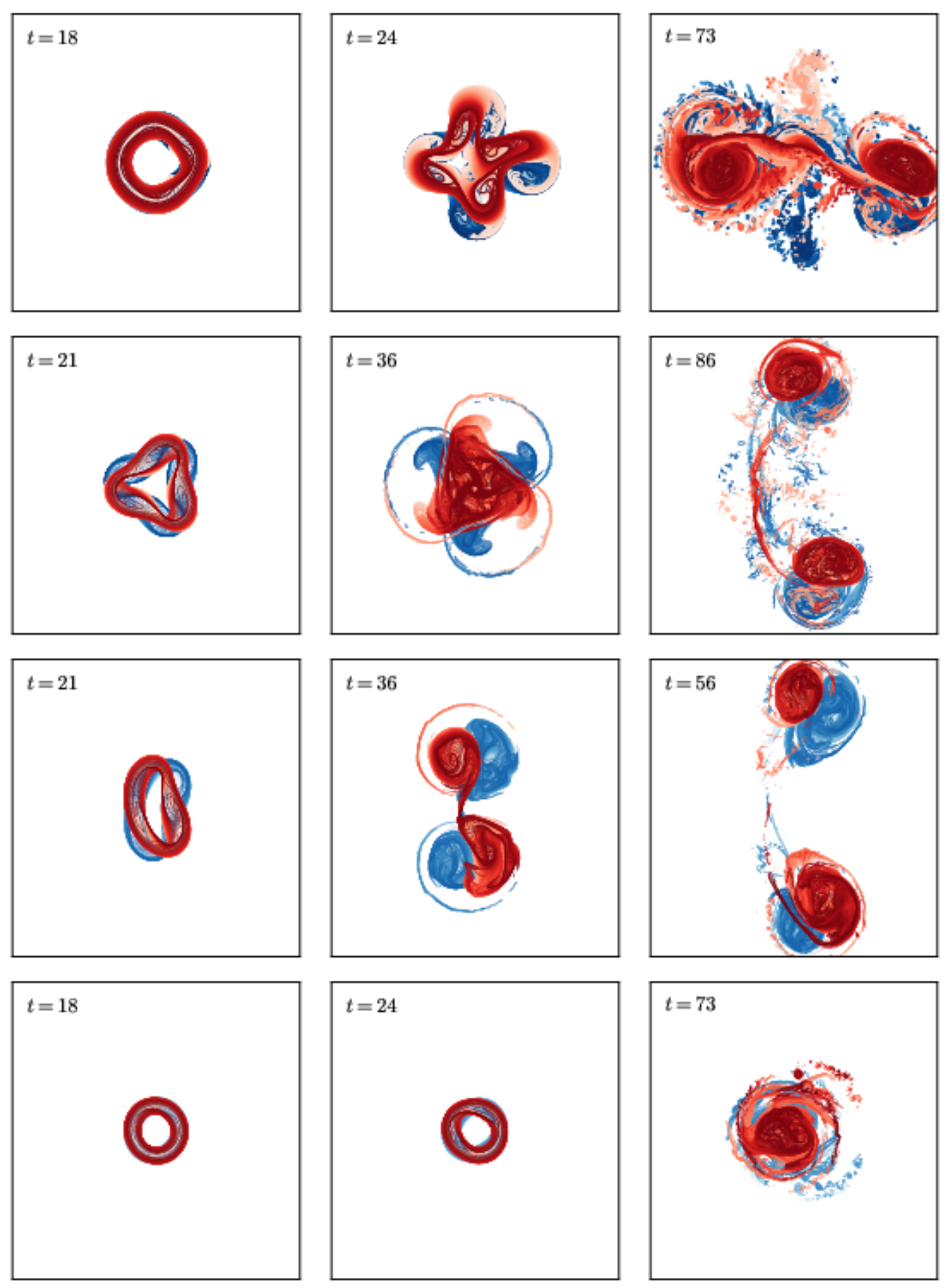

FIG. 3. Top view on the vortex bounding contours for $R_{0} / r_{0}=3$. Top row: $\ell_{z}=0$ and at times, from left to right, $t=18,24,73$. Second row: $\ell_{z}=13 / 51$, and $t=21,36,86$. Third row: $\ell_{z}=25 / 51$, and $t=21,36,56$. Bottom row: $\ell_{z}=1$, and $t=18,24,73$. Red contours correspond to positive PV while blue contours correspond to negative PV . 

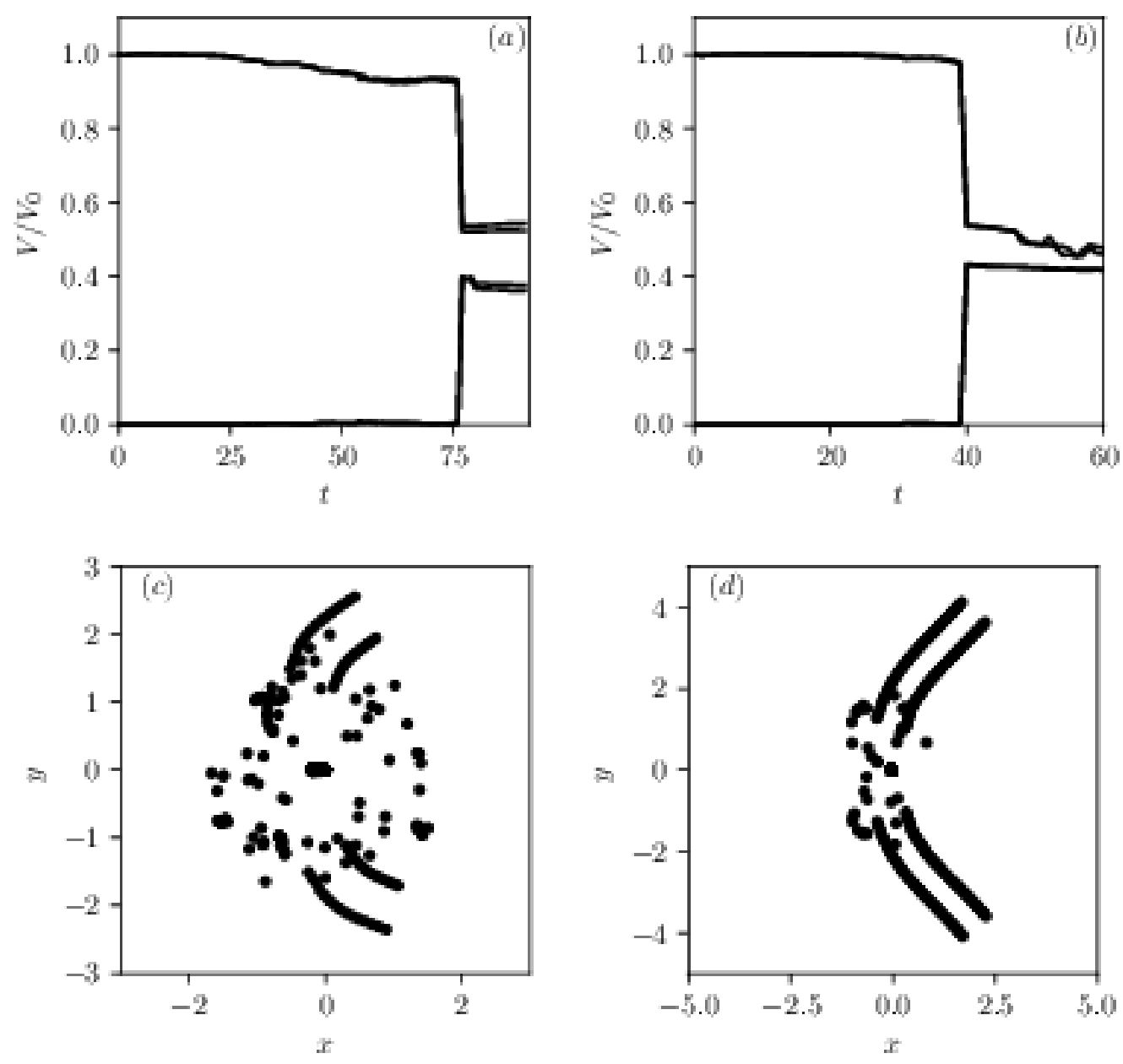

FIG. 4. Evolution of the volume of the 4 largest vortices, normalised by the volume of a single torus at $t=0$, for $R_{0} / r_{0}=3$, and $(a): \ell_{z}=13 / 51$ and $(b): \ell_{z}=25 / 51$. Trajectories of the vortex centres for $(c): \ell_{z}=13 / 51$ and $(d): \ell_{z}=25 / 51$. The location of the centres are marked by $\bullet$ every $\Delta t=1$.

(a) and (b) of Fig. 4 shows that the tori each split into two main secondary structures at $t \simeq 77$ for $\ell_{z}=13 / 51$ and $t \simeq 40$ for $\ell_{z}=25 / 51$. The split is asymmetric, with one vortex containing about $50 \%$ of the PV on the initial torus, while the second one carries about $40 \%$ or less of the volume. This asymmetry explains the curvature of the trajectory of the hetons observed on the panels $(c)$ and $(d)$ of Fig. 4. The scattering of points near the centre is the trace of filaments which are generated during the early evolution of the flows, before the four main secondary vortices are fully formed.

For $R_{0} / r_{0} \ell_{z}=1$, the two opposite-signed vortices formed at the centre of the domain 

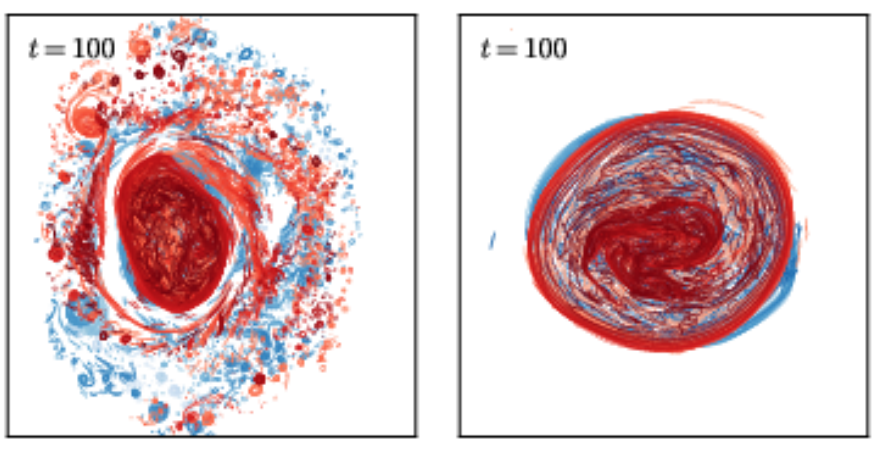

FIG. 5. Top view on the vortex bounding contours for (left) $\ell_{z}=1$ and $R_{0} / r_{0}=4$, and (right) $\ell_{z}=25 / 51$ and $R_{0} / r_{0}=2$ at time $t=100$.

interact only weakly and are meta-stable. The late evolution is somehow similar to the one of compact hetons when the vortices are sufficiently offset in the vertical direction. ${ }^{6}$ This is also representative of all interactions for tori sufficiently thick and/or sufficiently separated in the vertical direction. Further examples are given in Fig. 5 for $\ell_{z}=25 / 51$ and $R_{0} / r_{0}=2$, a case where the tori are thicker than the previous case and moderately vertically offset, and for $\ell_{z}=1$ and $R_{0} / r_{0}=4$, a case where the tori are thinner but still well separated in the vertical direction.

Even when the final state consists mainly of a single pair of hetons, the early evolution of the flow can still be complex, as shown in Fig. 6 for $\ell_{z}=25 / 51$ and $R_{0} / r_{0}=5$. In this case the tori are sensitive to high wavenumber modes. Modes $m=4$ and $m=5$ clearly develop on the tori (see first panel of Fig. 6). Four main vortices with unequal volumes form for each torus (second panel of Fig. 6). The like-signed vortices are close together and eventually merge to form back single, distorted, vortices both at the top and at the bottom. These two opposite-signed vortices tear each other apart and form a pair of hetons, with a large number of debris and small scale secondary vortices.

\section{B. Thin tori, $R_{0} / r_{0} \geq 7.2$}

We next consider thinner tori. We first compare the evolution of tori of a given aspect ratio $R_{0} / r_{0}$ for four values of the vertical gap $\ell_{z}$. The evolution of the flow is presented in Fig. 7 for $R_{0} / r_{0}=7.2$. For $\ell_{z}=0$ (see the top row of Fig. 7), several modes compete. The early 

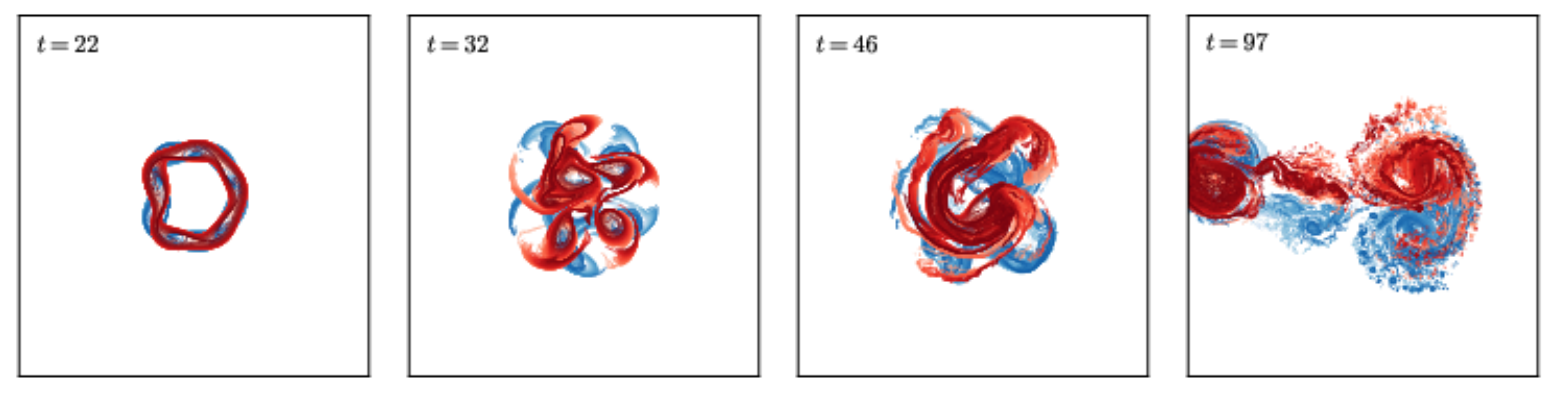

FIG. 6. Top view on the vortex bounding contours for $\ell_{z}=25 / 51, R_{0} / r_{0}=5$ at time $t=$ $22,32,46,97$.

evolution of the instabilities shows the simultaneous growth of modes $m=4,6,7,8$. Then seven coherent structures start to from from the re-organisation of the tori. The pattern is however irregular due to the fact that the mode $m=7$ competes with other modes. Partially due to this irregularity, some of these structures form very close to each other and merge. Nearer the end of the simulation five main hetons have formed, one being much larger than the others. This larger vortex however appears to be a compound vortex which later breaks into secondary structures.

There is an angular offset between the deformation of the two tori and the subsequent formation of the vortices. This is typical of the influence of baroclinic modes. This offset creates a dipolar moment. The vortices within each baroclinic pair are vertically misaligned, forming tilted hetons which self-propagate. On the contrary, in the case of two unstable likesigned tori the pairs of vortices form on the top of each other as shown in Appendix A.

When increasing $\ell_{z}$, while keeping $R_{0} / r_{0}$ the same, the predominance of the mode $m=5$ is stronger. In these cases, the flow evolution is dominated by the formation of five hetons. It should however be noted that PV distributions are not 5-fold symmetric, due to the presence and growth of of secondary modes with $m \neq 5$. In all cases the tori nonetheless self-organise into hetons which move away from the centre of the domain. Only PV debris and filaments, which have dynamically little importance, are left near the centre of the domain. For $\ell_{z}=0$ and $R_{0} / r_{0}=6.4$ (results not shown) the results are similar and the late evolution of the flow results in the formation of five hetons, with a more regular pattern than for $\ell_{z}=0$ and $R_{0} / r_{0}=7.2$. This is due to the predominance of the mode $m=5$ in this case. For the same gap, $\ell_{z}=0$, and slightly thinner tori, $R_{0} / r_{0}=8$ (result not shown), 

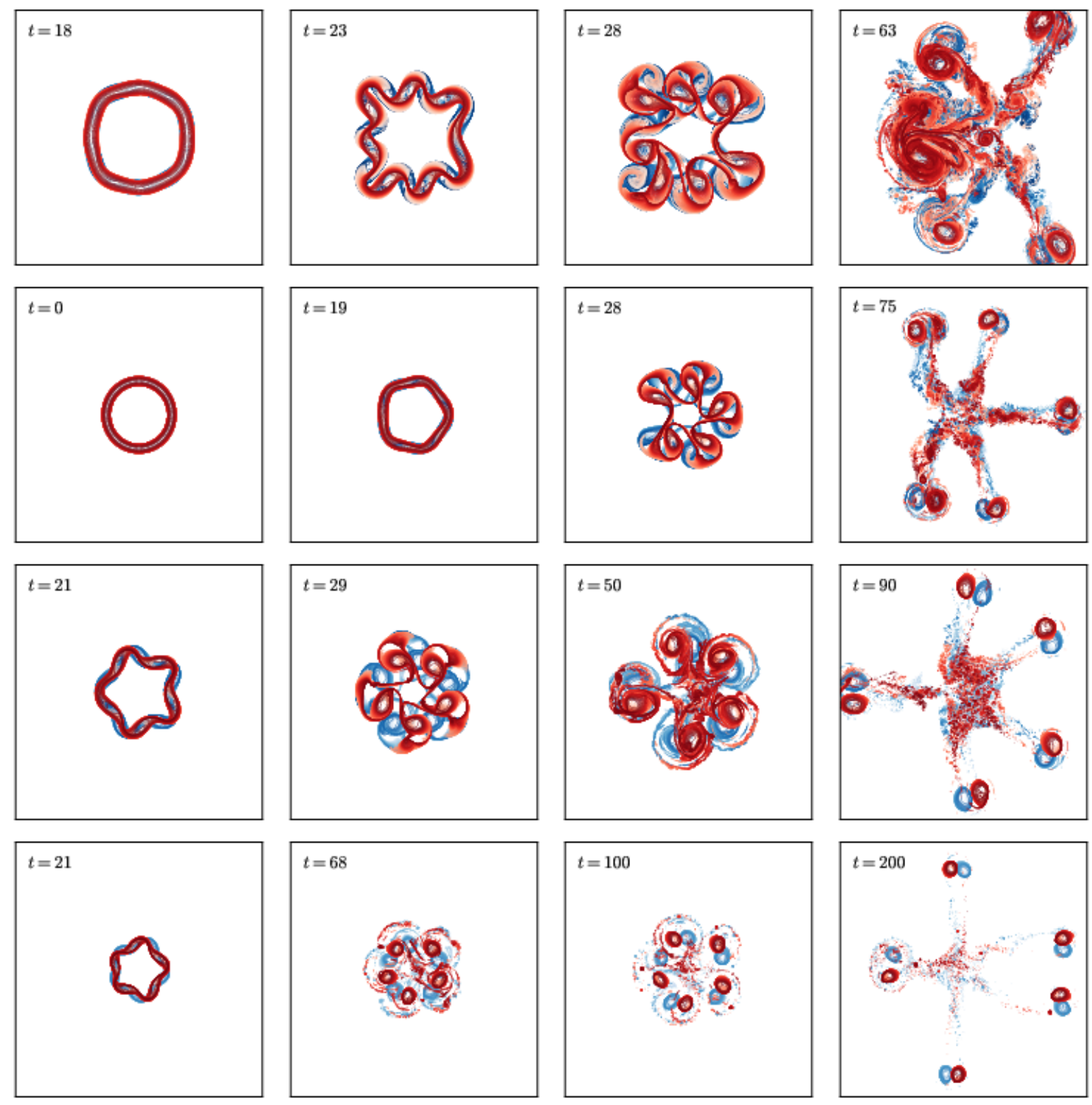

FIG. 7. Top view on the vortex bounding contours for $R_{0} / r_{0}=7.2$. Top row: $\ell_{z}=0$, at time $t=18,23,28,63$. Second row: $\ell_{z}=13 / 51, R_{0} / r_{0}=7.2$ at $t=0,19,28,75$. Third row: $\ell_{z}=25 / 51, R_{0} / r_{0}=7.2$ at $t=21,29,50,90$. Bottom row: $\ell_{z}=1, R_{0} / r_{0}=7.2$ at time $t=21,68,100,200$

the late evolution of the flow results in the formation of six hetons. Some of them are highly distorted and may further break. This intense deformation of the vortices has also been observed during the evolution of hetonic filaments. ${ }^{36}$. The strong interaction between the lower anticyclone and the upper cyclones can partially strain out the vortices. 

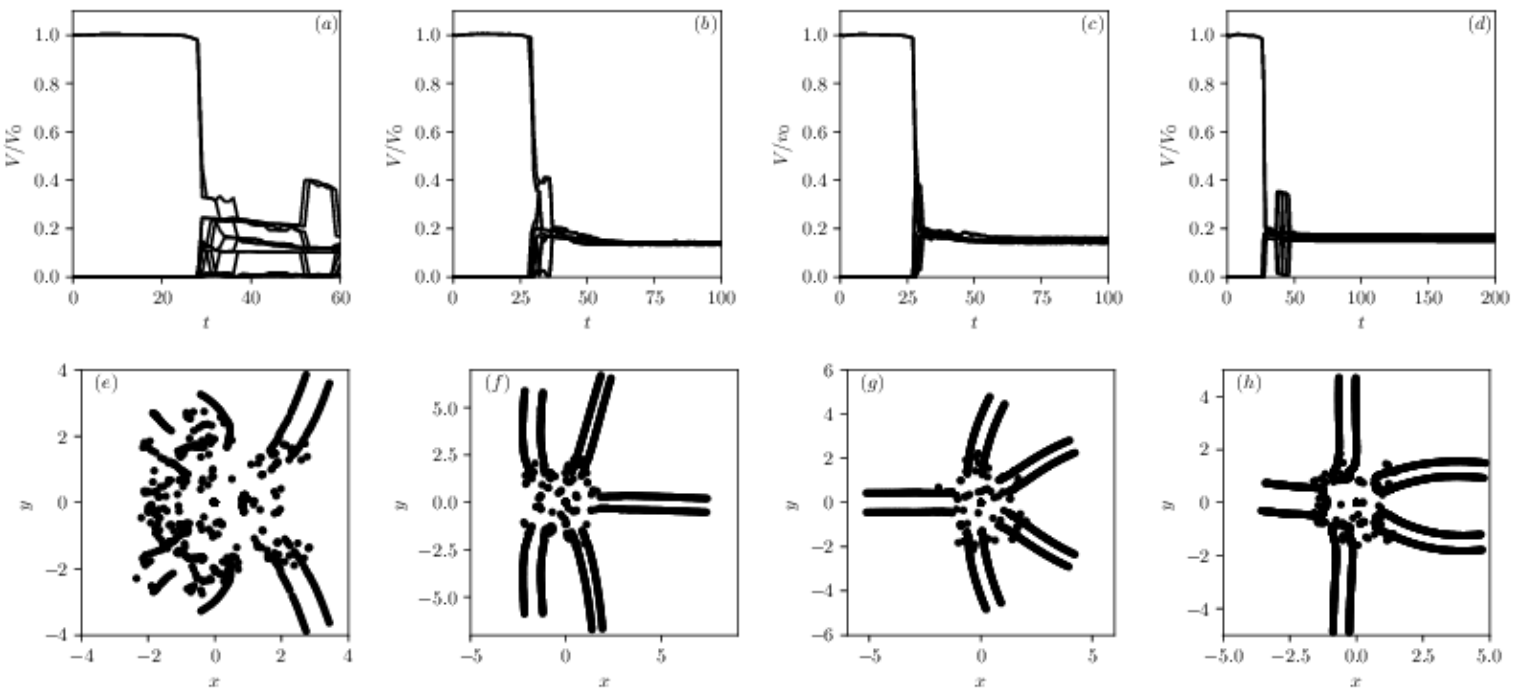

FIG. 8. Top row: evolution of the volume of the 10 largest vortices for $R_{0} / r_{0}=7.2$ and $(a): \ell_{z}=0$, (b): $\ell_{z}=13 / 51,(c): \ell_{z}=25 / 51,(d): \ell_{z}=1$. Bottom row trajectories of the centres of the 10 largest vortices for $R_{0} / r_{0}=7.2$ and $(e): \ell_{z}=0,(f): \ell_{z}=13 / 51,(g): \ell_{z}=25 / 51$ and $(h): \ell_{z}=1$.

Figure 8 shows the evolution of the volume of the ten largest vortices identified, as well as the trajectory of their centres, for $R_{0} / r_{0}=7.2$ and $\ell_{z}=0,13 / 51,25 / 51,1$. For $R_{0} / r_{0}=7.2$ and $\ell_{z}=0$, the evolution of the volume confirms the breaking of the tori into many secondary vortices from $t \simeq 29$. Then the evolution of the vortices volume shows a series of further vortex breaking and vortex merger. For larger $\ell_{z}$, the situation appears simpler with the formation of ten vortices carrying roughly a fifth of the volume of the individual tori. The small asymmetries between the vortices induce a small curvature in their trajectories, as seen from the bottom row of Fig. 8 .

We next repeat the numerical experiments for $R_{0} / r_{0}=12$ and $\ell_{z}=0,13 / 51,25 / 51,1$. The evolution of the flow is shown in Fig. 9. For $\ell_{z}=0$ (see the top row of Fig. 9), the mode $m=10$ dominates the flow evolution. Each torus self-organises into an irregular array of ten vortices. These vortices strongly interact with the vortices of the other torus and form hetons which start to move away from the centre of the domain. It should be noted that in this case again, the closeness of the opposite-signed PV vortices induces intense vertical and horizontal shear. This shear partially strains out the vortices which leave behind large quantities of filamentary PV and debris. For $\ell_{z}=13 / 51$ (see the second row of Fig. 9), eight hetons are formed. But, as seen from the early deformation of the tori, the evolution 

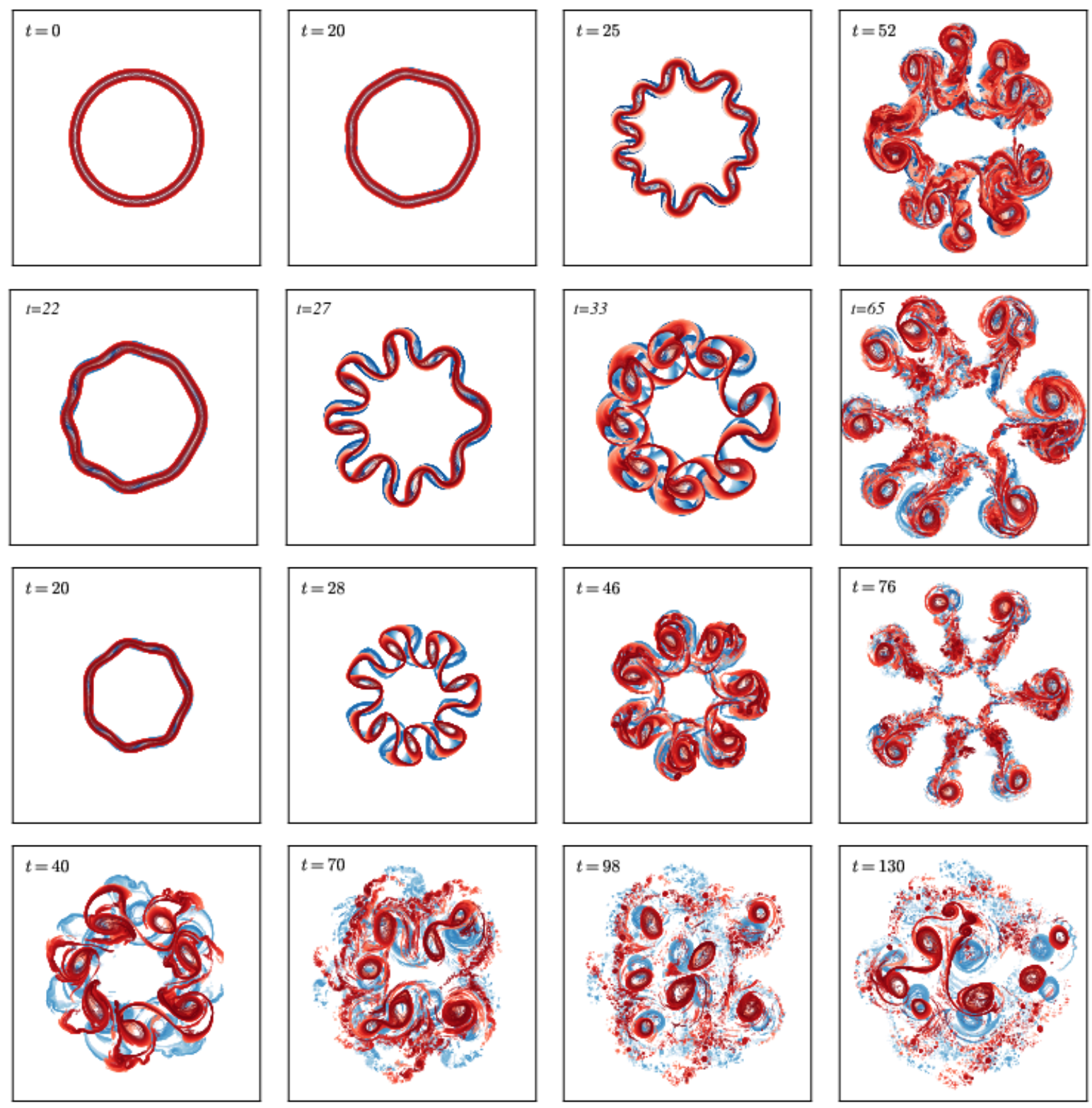

FIG. 9. Top view on the vortex bounding contours for $R_{0} / r_{0}=12$. Top row: $\ell_{z}=0$, at time $t=0,20,25,52$. Second row: $\ell_{z}=13 / 51$ and $t=22,27,33,65$. Third row $\ell_{z}=25 / 51$ and $t=20,28,46,76$. Bottom row $\ell_{z}=1$, and $t=40,70,98,130$.

is asymmetric. For $\ell_{z}=25 / 51$, (see the third row of Fig. 9) the pair of tori self-organises into seven hetons, and the array of hetons is more regular. The situation changes when the tori are further separated in the vertical direction. Indeed, for $\ell_{z}=1$, the later evolution of the flow no longer consists of hetons moving away from the centre. Instead, we see a convoluted motion of vortices near the centre of the domain. Within the same vertical level, 
like-signed vortices strongly interact and some partially merge. It should be noted that the linear stability analysis of a regular circular array of seven like-signed vortices is unstable in QG. ${ }^{42}$. The convoluted motion observed for $\ell_{z}=1$ is, in part, due to the weakening of the coupling between the lower anticyclones and upper cyclones. For small vertical gaps, the interaction between the cyclones and the anticyclones is strong enough to force the vortices to move away as baroclinic pairs. For $\ell_{z}=1$, this interaction is too weak to counteract the chaotic horizontal motion of the like-signed vortices. This does not mean that some of the opposite-signed vortices do not form baroclinic dipoles. In fact, the two pairs of such dipole are seen to temporarily exist, see the two vortex pairs on the right on the bottom-right panel of Fig. 9 for $t=130$. However, these two hetons are on the course to collide and the two lower anticyclonic vortices merge by $t \simeq 141$.

The evolution of the flow is further described by the evolution of the volume of the 16 largest identified vortices in the flow (20 largest vortices for the case $\ell_{z}=0$ ) as well as the trajectory of the vortex centres. Results are presented in Fig. 10. For $\ell_{z}=0$, results confirm that the vortices formed by the destabilisation of the tori have different volumes creating asymmetries in the flow. The evolution of the vortices' volume also shows episodes of strong interactions whereby some vortices merger and large filaments of PV detach from the main vortices. For the intermediate gaps $\ell_{z}=13 / 51$ and $\ell_{z}=25 / 51$ the evolution is less convoluted with fewer events of strong interaction. Moreover, in these cases, the events are short-lived. The main secondary vortices have a typical volume slightly less than a seventh of the initial tori. This shows that a larger quantity of debris and filaments has been generated, compared to the case with $R_{0} / r_{0}=7.2$. Finally the top-right panel of Fig. 10 confirms the convoluted dynamics for $\ell_{z}=1$. Strong vortex interactions such as vortex merger take place. The bottom panels of Fig. 10 shows the trajectory of the vortex centres. The motion of the hetons away from the centre is hinted for $\ell_{z}=0$, and clearly shown for the intermediate gaps $\ell_{z}=13 / 51$ and $\ell_{z}=25 / 51$. On the contrary the trajectories of the vortex centres for $\ell_{z}=1$ are complex. Recall that if a vortex splits into secondary vortices, or if two vortices merge, the vortex centres are redefined and therefore change location: hence the location of the centres appears more scattered.

Overall, this phenomena also show strong similarities with the expulsion of vortex dipoles by the destabilisation of pairs of two-dimensional, concentric, uniform-PV rings with zero net circulation on the $f$-plane ${ }^{43}$, and on the $\beta$-cone. ${ }^{34,35}$. 

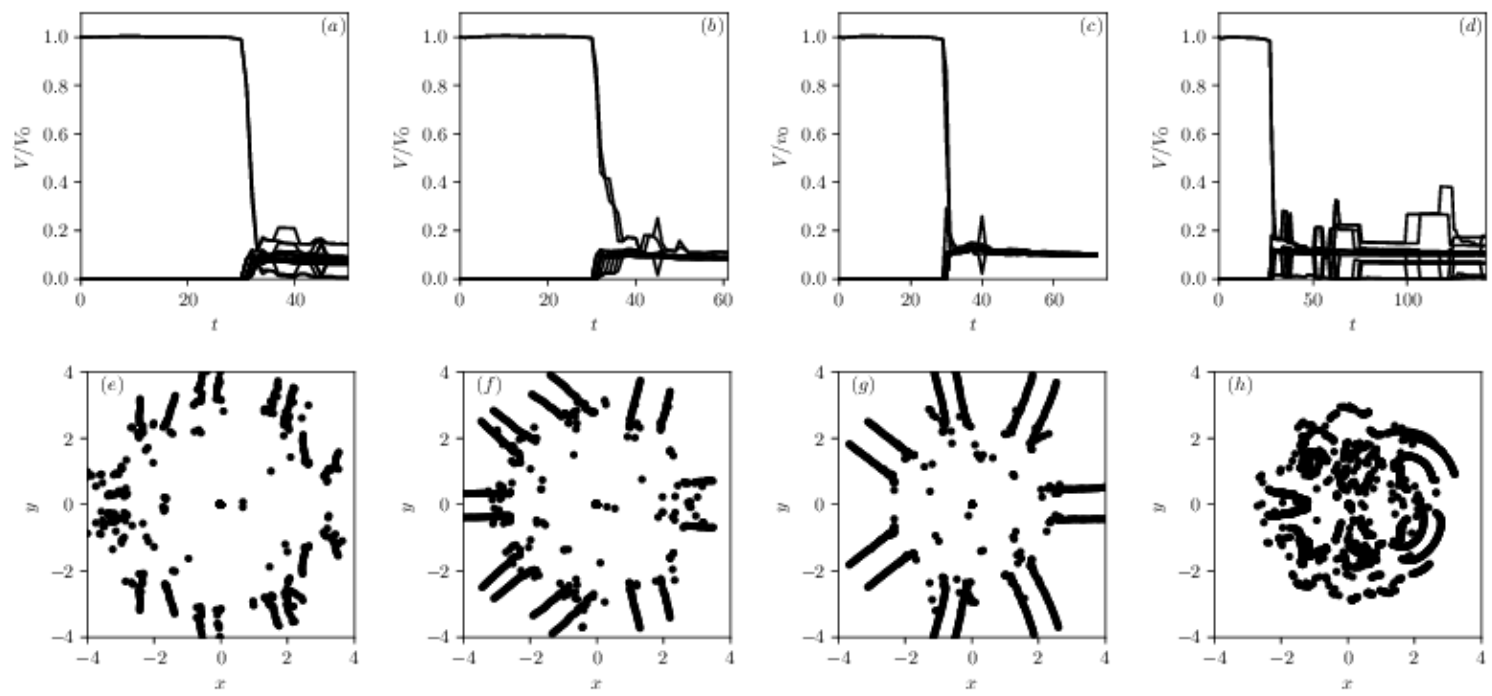

FIG. 10. Top row: evolution of the volume of the 16 largest vortices for $R_{o} / r_{o}=12$ and $(a): \ell_{z}=0$ (20 largest shown in this case), $(b): \ell_{z}=13 / 51,(c): \ell_{z}=25 / 51$, and $(d): \ell_{z}=1$. Bottom row: trajectories of the centre of the 16 largest vortices for $R_{o} / r_{o}=12$ and $(e): \ell_{z}=0,(f): \ell_{z}=13 / 51$, $(g x): \ell_{z}=25 / 51$, and $(h): \ell_{z}=1$.

\section{Fourier analysis of the central contour deformation}

In order to better understand the factors leading to excitation of higher unstable modes, we further describe the early evolution of the flow by studying the evolution of the Fourier decomposition of the deformation the contours bounding the tori. To that purpose, we first extract a contour bounding a torus. The contours are described by a collection of $n_{p}$ nodes $\left(x_{i}, y_{i}\right)$ which are advected in a Lagrangian way during the nonlinear simulation. We first determine their polar coordinates $\left(\theta_{i}, r_{i}\right)_{1 \leq i \leq n_{p}}$, where $\theta_{i}=\tan ^{-1}\left(y_{i} / x_{i}\right)$ and $r_{i}=\sqrt{x_{i}^{2}+y_{i}^{2}}$. The contours are then re-sampled, in the post-treatment procedure, by a new collection of points $\left(\tilde{\theta}_{j}, \tilde{r}_{j}\right)_{1 \leq j \leq N_{p}}$ equally-spaced in the azimuthal direction, $\tilde{\theta}_{j}=2 \pi(j-1) / N_{p}$. We use $N_{p}=1024$. The local radius $\tilde{r}_{j}$ is obtained by local quadratic polynomial interpolation from the known collection $\left(\theta_{i}, r_{i}\right)_{1 \leq i \leq n_{p}}$. We then perform an FFT on the signal $\left(\tilde{\theta}_{j}, \tilde{r}_{j}\right)_{1 \leq j \leq N_{p}}$. The first Fourier coefficient gives the mean radius $r_{\text {mean }}$ of the contour and is ignored in the analysis below. We only discuss the radial departure $r^{\prime}$ of the contours from the mean circle. It should be noted that this analysis can be repeated for any contour as far as the the contour does not overturn and $\tilde{r}_{j}$ remains uniquely defined for any angle $\tilde{\theta}_{j}$. 
TABLE II. Growth rates for $R_{0} / r_{0}=12$ and $\ell_{z}=0$ and $\ell_{z}=25 / 51$

\begin{tabular}{|c|c|c|c|}
\hline \multicolumn{2}{|c|}{$\ell_{z}=0$} & \multicolumn{2}{|c|}{$\ell_{z}=25 / 51$} \\
\hline$\sigma_{r} / q \times 10^{-2}$ & $m$ & $\sigma_{r} / q \times 10^{-2}$ & $m$ \\
\hline 9.423 & 13 & 7.964 & 10 \\
\hline 9.363 & 14 & 7.834 & 9 \\
\hline 9.353 & 12 & 7.780 & 11 \\
\hline 9.195 & 15 & 7.417 & 8 \\
\hline 9.130 & 11 & 7.257 & 12 \\
\hline 8.954 & 16 & 6.741 & 7 \\
\hline 8.739 & 10 & 6.361 & 13 \\
\hline 8.670 & 17 & 5.840 & 6 \\
\hline 8.368 & 18 & 5.047 & 14 \\
\hline 8.177 & 9 & 4.754 & 5 \\
\hline
\end{tabular}

Recall that no perturbation is initially imposed on the tori. Perturbations only arise from the low amplitude numerical noise. Table II provides the list of the normalised growth rates $\sigma_{r} / q$ for the ten most amplified modes and their associated wavenumber $m$ for $R_{0} / r_{0}=12$, $\ell_{z}=0$ and $\ell_{z}=25 / 51$. We see that several modes have very similar growth rates.

Figure 11 shows the evolution of the norm of the complex Fourier coefficients $\left|\hat{r}^{\prime}\right|$ for the radial departure of the contour for $R_{0} / r_{0}=12$, and $\ell_{z}=0$ and $\ell_{z}=25 / 51$. For $\ell_{z}=0$ we first analyse the outer contours for both tori in their mid-section. By construction, these contours correspond, at $t=0$ to circles of radius $R_{0}+r_{0}$. We also analyse the two outer contours where the two tori 'touch': i.e. the top outer bounding contour of the lower vortex and the bottom outer bounding contour of the upper torus. Results are presented in the top and second rows of Fig. 11. At early times (not shown) the main deformation results from the vertical shear. The contours are displaced horizontally, non-uniformly with height, corresponding to an azimuthal mode $m=1$.

Low wavenumber deformation modes are excited and the mode $m=7$ starts to develop. Recall that perturbations are purely stemming from numerical noise and therefore depend on the discretisation, see AppendixB. ${ }^{1}$ It should be noted that modes $m=8,9$, and 7 are the

\footnotetext{
${ }^{1}$ The perturbation, from discretisation error, of an unstable mode with wavenumber $m$ may be favoured
} 
most amplified modes for a single torus of aspect ratio $R_{0} / r_{0}=12 .{ }^{32}$ We can however see the rapid amplification of several higher wavenumber modes as higher wavenumber perturbations appear in the flow. The amplification appears larger in the region where the two tori touch. This is consistent with high wavenumber baroclinic modes which are often localised in the regions where the vertical shear is maximum. ${ }^{36}$ Overall, the norms of the Fourier coefficients are nearly twice as large in the region where the tori touch compared to the tori mid-section, stressing the effect of the vertical shear on the growth of the deformation. Results are similar for $\ell_{z}=25 / 51$. In this case there are fewer high wavenumber modes, which is consistent with the linear stability analysis. Separating the tori in the vertical direction weakens the high wavenumber baroclinic modes.

Figure 12 provides snapshots of the results from the Fourier analysis for $\ell_{0}$ and various additional aspect ratios $R_{0} / r_{0}=3,6.4,7.2,8$. This confirms that low wavenumber modes grow preferably on thick tori. Finally Fig. 13 gives the results of the Fourier analysis for $R_{0} / r_{0}=12$ and various vertical gaps $\ell_{z}=0,13 / 51,25 / 51,1$. The results also confirm the tendency that high wavenumber modes grow preferably when the vertical shear is intense. This is the case for small vertical gaps between the tori.

\section{CONCLUSIONS}

We have investigated the formation of hetons stemming from the destabilisation of a pair of tori of opposite-sign uniform PV. The tori are sensitive to both barotropic and baroclinic instabilities due to the presence of both horizontal and vertical shear. The tori self-organise into arrays of secondary vortices. The flow evolution depends on the aspect ratio $R_{0} / r_{0}$ of the PV tori as well as the gap $\ell_{z}$ separating them in the vertical direction: thin tori which are close to each other in the vertical direction are more sensitive to instabilities with high azimuthal wavenumbers. Thick tori tend first to self-organise into compact and/or elongated structures near the centre of the domain. Then these structures break into (typically) a pair of hetons. This is similar to the evolution of equivalent compact cylindrical hetons. ${ }^{6,8,10}$ Thin tori produce large numbers of secondary vortices. The number of vortices depends not only on the tori aspect ratio and the vertical gap but also on the perturbations exciting the instabilities since the tori are typically sensitive to a collection of modes with different

of the number of nodes discretising the contours is a multiple of $m$. 

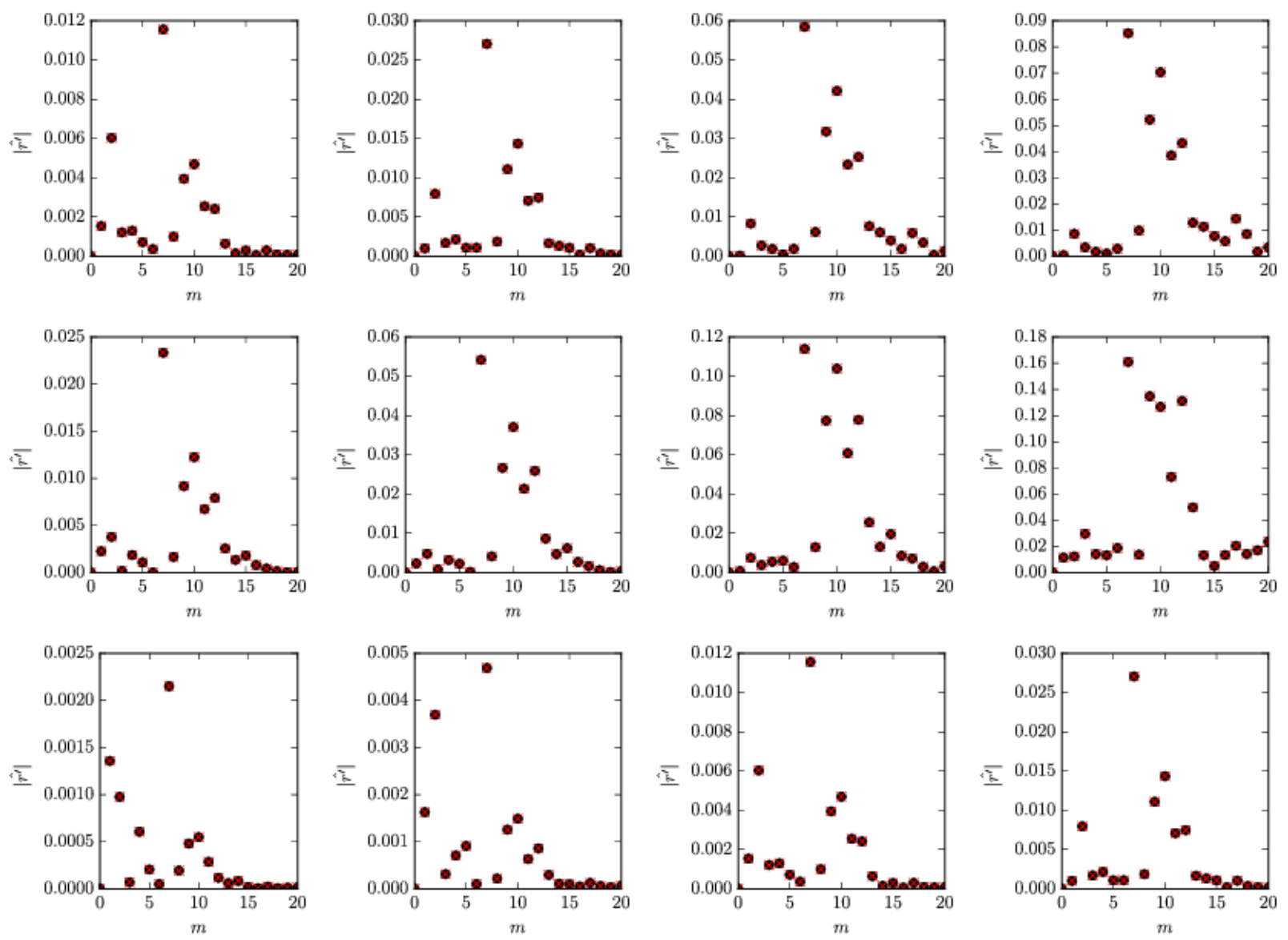

FIG. 11. Norm of the complex Fourier coefficients for the deformation of the central outer bounding contours for both tori for $R_{0} / r_{0}=12$. Top row: $\ell_{z}=0$ at $t=19,21,23,24$. Second row: same as top row but for the outer top (resp. bottom) contour for the lower (resp. upper) torus. Bottom row: $\ell_{z}=25 / 51$ at $t=15,171921$. $\bullet$ indicates results for the lower vortex while $\times$ indicates results for the upper vortex.

azimuthal wavenumbers, but with similar growth rates. In our simulations, perturbations stem from numerical noise. This noise is however not a white noise and its spectrum depends on the spatial resolution. The vortices, formed from one of the tori, pair with the closest opposite-signed vortex formed from the other torus. They form a baroclinic dipole, known as a heton or modon The orientation of the dipolar moment of the heton makes it selfpropagate outwards, away form the region of formation, transporting with it momentum and mass. The re-organisation of the tori of PV provides an elementary mechanism to produce hetons in a rapidly rotating, stably stratified environment. 

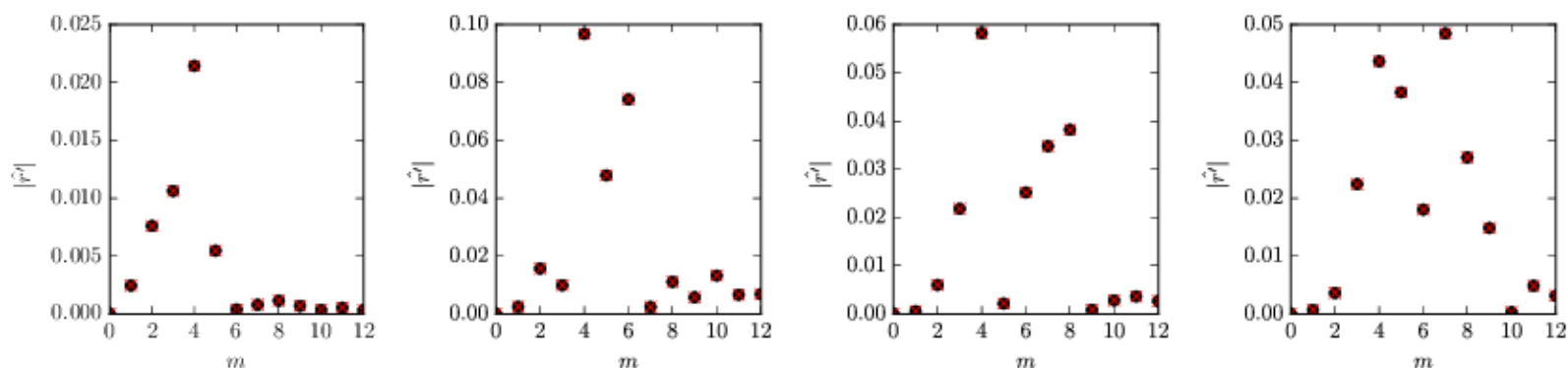

FIG. 12. Norm of the complex Fourier coefficients for the deformation of the central outer bounding contours for $\ell_{z}=0,\left(R_{0} / r_{0}, t\right)=(3,19),(6.4,23),(7.2,21),(8,21)$.
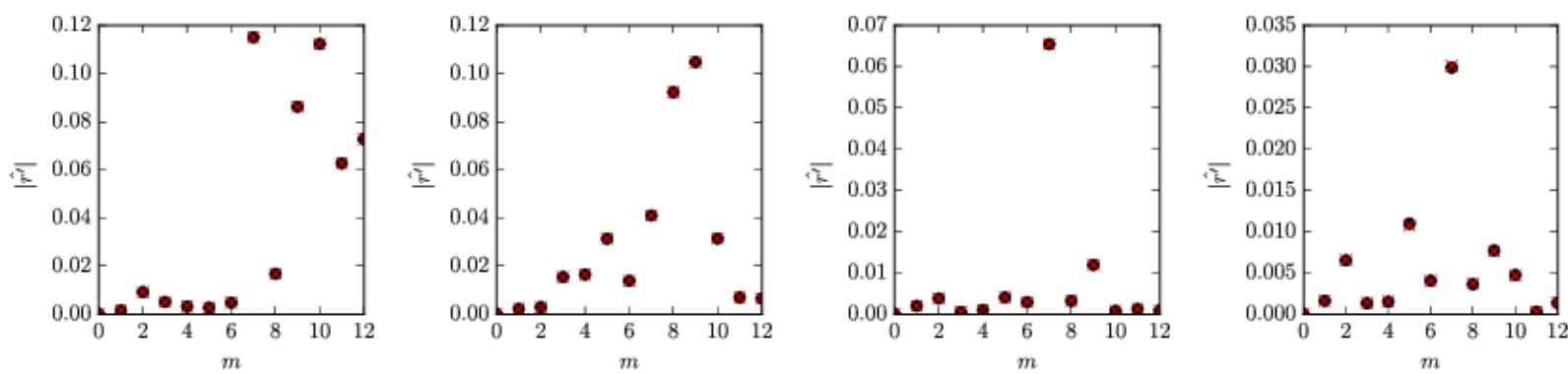

FIG. 13. Norm of the complex Fourier coefficients for the deformation of the central outer bounding contours for $R_{0} / r_{0}=12,\left(\ell_{z} / r_{0}, t\right)=(0,25),(13 / 51,25),(25 / 51,21),(1,19)$.

We have however seen that when a large number of vortices is formed and when the vertical coupling between the opposite-signed vortices is weak, the flow exhibits a complex, chaotic dynamics. This restricts the self-propagation of the vortices. The formation of robust hetons from thin tori necessitates a strong vertical coupling between opposite-signed vortices. This coupling must be strong enough to dominate the other horizontal interactions between the like-signed vortices.

This study has restricted attention to the QG dynamics. This is relevant to most mesoscale vortices in the oceans. For submesoscale vortices, ageostrophic effects become important. Cyclonic and anticyclonic vortices exhibit different dynamical properties as well as different stability properties. The importance of submesoscale processes in the energy budget of the oceans is now recognised. As mentioned above, hetons provide an effective mechanism to transport heat, mass and momentum over large distance in the oceans. Hence the study of the dynamics of hetons as well as their formation mechanisms must also be 

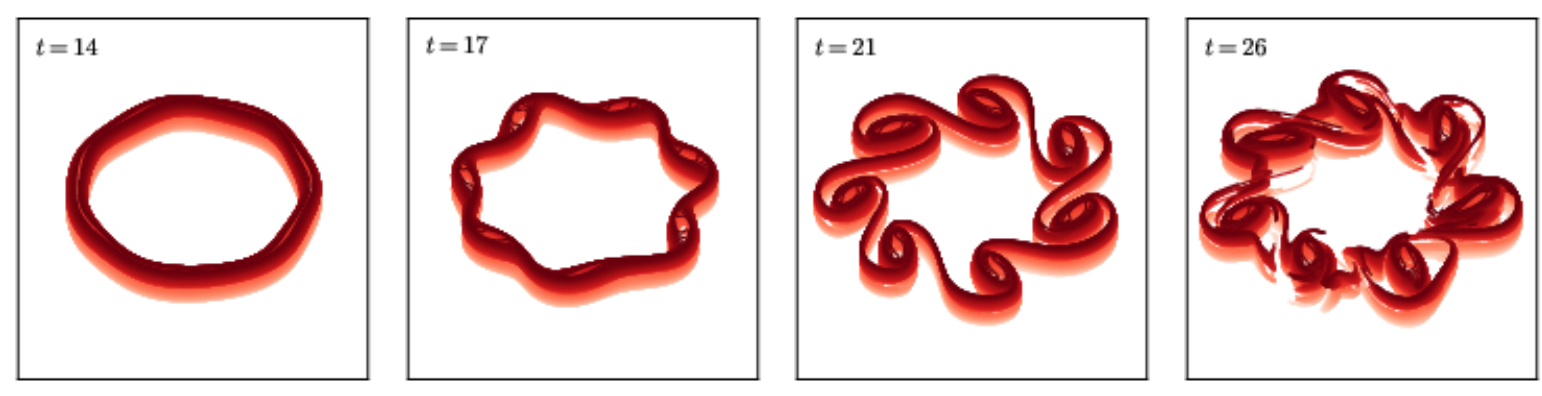

FIG. 14. Top view on the vortex bounding contours for $\ell_{z}=0, R_{0} / r_{0}=12$ at time $t=$ 14, 17, 20, 26 for like-signed tori. Vortices are viewed orthographically at an angle to $45^{\circ}$ from the vertical axis. The contrast in colour indicates the depth of the contours: lighter contours are near the bottom.

better understood at smaller scales where ageostrophic effects are no longer negligible. This is a natural extension of the present study.

Data are available on request from the author.

\section{Appendix A: Like-signed tori}

We show here that the angular offset between the deformation of the tori, hence the formation of vortices, is specific to the situation where the tori have opposite PV as discussed in section IV B. We repeat one of our experiments with $\ell_{z}=0$ and $R_{0} / r_{0}=12$ but for like-signed tori. Both tori have uniform PV $q=2 \pi$. Again, no external perturbation is introduced. Modes grow from the background low amplitude numerical noise. Results, presented in Fig. 14, show that the vortices form on top of each other and that the evolution of both tori is similar.

\section{Appendix B: Contour resolution}

We present an alternative simulation for a pair of opposite-signed uniform PV tori with $\ell_{z}=0$ and $R_{0} / r_{0}=12$ presented in section IV B. Here, we only consider the early evolution of the flow, and we fix the number of nodes used to discretised all vortex bounding contours (no surgery permitted). To avoid exciting specific azimuthal modes by using the number

of equally-spaced nodes proportional to one of the unstable wavenumbers, each contour is 

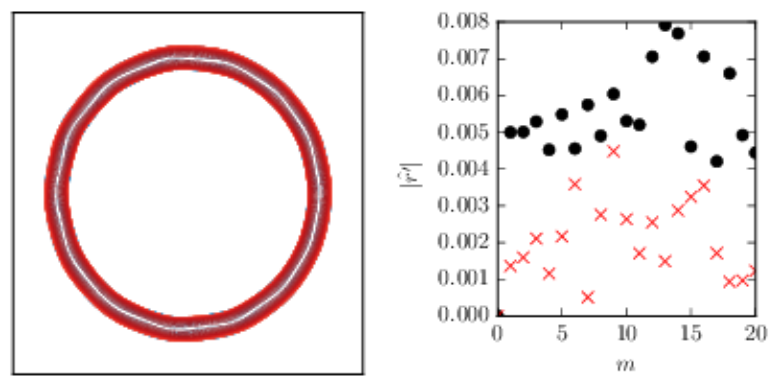

FIG. 15. Left: top view on the vortex bounding contours for $R_{0} / r_{0}=12, \ell_{z}=0$ at $t=49$. Right: norm of the complex Fourier coefficients at $t=49$. The vortex bounding contours mapped by a prime number of nodes.

discretised by a prime number of nodes: $n_{p}=317$. Note that there is no simple way to impose this constraint in general for the long term evolution of the flow. The number of nodes used to discretise a contour depends on its length and on the local curvature, and normally varies from one contour to the other. It also varies with time for any given contour as it deforms. A snapshot at $t=49$ is shown in Fig. 15. It should be noted that using a prime number of nodes has delayed the development of the instability due to the reduction of the numerical noise a the unstable wavenumbers. This also allows the most unstable higher frequencies to develop.

\section{REFERENCES}

${ }^{1}$ V. Gryanik, "Dynamics of singular geostrophic vortices in a two-level model of the atmosphere (or ocean)," Izv. Atmos. Ocean Phys. 19, 171-179 (1983b).

${ }^{2} \mathrm{~V}$. Gryanik, "Dynamics of localized vortex perturbations 'vortex charges' in a baroclinic fluid," Izv. Atmos. Ocean Phys. 19, 347-352 (1983a).

${ }^{3}$ N. Hogg and H. Stommel, "The heton, an elementary interaction between discrete baroclinic geostrophic vortices and its implications concerning eddy heat flow," Proc. R. Soc. Lond. A. 397, 1-20 (1985).

${ }^{4}$ G. R. Flierl, "On the instability of geostrophic vortices," J. Fluid Mech. 197, 339-388 (1988). 
${ }^{5}$ K. R. Helfrich and U. Send, "Finite-amplitude evolution of two-layer geostrophic vortices," J. Fluid Mech. 197, 331-148 (1988).

${ }^{6}$ J. N. Reinaud and X. Carton, "The stability and the nonlinear evolution of quasigeostrophic hetons," J. Fluid Mech 636, 109-135 (2009).

${ }^{7}$ J. N. Reinaud, "On the stability of continuously stratified quasi-geostrophic hetons," Fluid Dyn. Res. 47, 035510 (2015).

${ }^{8}$ V. G. Makarov, M. A. Sokolovskiy, and Z. Kizner, "Doubly symmetric finite core heton equilibria," J. Fluid Mech. 708, 397-417 (2012).

${ }^{9}$ B. Shteinbuch-Fridman, V. Makarov, and Z. Kizner, "Transitions and oscillatory regimes in two-layer geostrophic hetons and tripoles," J. Fluid Mech. 810, 535-553 (2017).

${ }^{10}$ Z. Kizner, D. Berson, and R. Khvoles, "Baroclinic modon equilibria on the beta-plane: stability and transitions," J. Fluid Mech. 468, 239-270 (2002).

${ }^{11}$ W. Young, "Some interactions between small numbers of baroclinic, geostrophic vortices," Geophys. and Astrophys. Fluid Dyn 33, 35-61 (1985).

12Z. Kizner, "Stability and transitions of hetonic quartets and baroclinic modons," Phys. Fluids 18, 056601 (2006).

${ }^{13}$ M. A. Sokolovskiy and X. Carton, "Baroclinic multipole formation from heton interaction," Fluid Dyn. Res. 42, 045501 (2010).

${ }^{14}$ J. N. Reinaud and X. Carton, "Head on collisions between two quasi-geostrophic hetons in a continuously stratified fluid," J. Fluid Mech. 779, 144-180 (2015).

${ }^{15}$ J. N. Reinaud and X. Carton, "The interaction between two oppositely travelling, horizontally offset, antisymmetric quasi-geostrophic hetons," J. Fluid Mech. 795, 409-443 (2016).

${ }^{16}$ S.-Y. Chao and P.-T. Shaw, "Close interactions between two pairs of heton-like vortices under sea ice," J. Geophys. Res.: Oceans 104, 23591-23605 (1999).

${ }^{17}$ K. I. C. Oliver, D. P. Eldevik, T. ands Stevens, and A. J. Watson, "A Greenland sea perspective on the dynamics of postconvective eddies," J. Phys. Oceanogr. 38, 2755-2771 (2008).

${ }^{18}$ Y. Morel and J. C. McWilliams, "Effects of isopycnal and diapycnal mixing on the stability of oceanic currents," J. Phys. Oceanogr. 31, 2280-2296 (2001).

${ }^{19}$ L. Chérubin, X. Carton, and D. G. Dritschel, "Vortex dipole formation by baroclinic instability of boundary currents," J. Phys. Oceanogr. 37, 1661-1676 (2007). 
${ }^{20}$ G. F. Flierl, X. Carton, and C. Messager, Eur. Ser. Appl. Ind. Math. 7, 137-150 (1999).

${ }^{21}$ S. P. Meacham, "Meander evolution on piecewise-uniform, quasi-geostrophic jets." J. Phys. Oceanogr. 21, 1139-1170 (1991).

${ }^{22}$ J. M. Bane, L. M. O’Keefe, and D. R. Watts, "Mesoscale eddies and submesoscale coherent vortices: Their existence near and interactions with the gulf-stream," Mesoscale/Synoptic Coherent Structures in Geophysical Turbulence; Proceedings of the 20th International Liege Colloquium on Ocean Hydrodynamics. Elsevier, New York, 501-518 (1999).

${ }^{23}$ x. Carton, L. Chérubin, J. Paillet, Y. Morel, A. Serpette, and B. Le Cann, "Meddy coupling with a deep cyclone in the gulf of cadiz." J. Mar. Syst. 32, 13-42 (2002).

${ }^{24}$ N. Serra, I. Ambar, and R. Käse, "Observations and numerical modelling of the mediterranean outflow splitting and eddy generation," Deep-Sea Res. II 52, 383-408 (2005).

${ }^{25}$ T. A. D., D. W. Waugh, and S. D. Guzewich, "What causes Mars' annular polar vortices," Geophys. Res. Lett. 44, 71-78 (2017).

${ }^{26}$ W. J. Seviour, D. W. Waugh, and R. K. Scott, "The stability of Mars's annular polar vortex," J. Atmos. Sci. 74, 1533-1547 (2017).

${ }^{27}$ M. Rostami and V. Zeitlin, "Understanding dynamics of Martian winter polar vortex with "improved" moist-convective shallow water model," J. Phys.: Conf. Ser. 936, 012005 (2017).

${ }^{28}$ D. G. Dritschel, "Contour surgery: A topological reconnection scheme for extended integrations using contour dynamics," J. Comput. Phys. 77, 240-266 (1988).

${ }^{29}$ J. P. Kossin and W. H. Schubert, "Mesovortices, polygonal patterns and rapid pressure falls in hurricane-like vortices," J. Atmos. Sci. 58, 2196-2209 (2001).

${ }^{30}$ Y. G. Morel and X. J. Carton, "Multipolar vortices in two-dimensional incompressible flows," J. Fluid Mech. 267, 23-51 (1994).

${ }^{31}$ A. Adriani, A. Mura, G. Orton, C. Hansen, F. Altieri, M. L. Moriconi, J. Rogers, G. Eichstädt, T. Momary, A. P. Ingersoll, G. Filacchione, G. Sindoni, F. Tabataba-Vakili, B. M. Dinelli, F. Fabiano, S. J. Bolton, J. E. P. Connerney, S. K. Atreya, J. I. Lunine, F. Tosi, A. Migliorini, D. Grassi, G. Piccioni, R. Noschese, A. Cicchetti, C. Plainaki, A. Olivieri, M. E. O’Neill, D. Turrini, S. Stefani, R. Sordini, and M. Amoroso, "Cluster of cyclones encircling jupiter's poles," Nature 555, 216-219 (2018).

${ }^{32}$ J. N. Reinaud and D. G. Dritschel, "The stability and nonlinear evolution of quasigeostrophic toroidal vortices," J. Fluid Mech. 863, 60-78 (2019). 
${ }^{33}$ M. Sokolovskiy and J. Verron, Dynamics of vortex structures in a stratified rotating fluid (Springer, Cham Heildelberg New York Dordrecht London, 2014).

${ }^{34}$ M. Rabinovich, Z. Kizner, and G. Flierl, "Bottom-topography effect on the instability of flows around a circular island," J. Fluid Mech. 856, 202-227 (2018).

${ }^{35}$ M. Rabinovich, Z. Kizner, and G. Flierl, "Barotropic annular flows, vortices and waves on a beta cone," J. Fluid Mech. 875, 225-253 (2019).

${ }^{36}$ J. N. Reinaud, "Stability of filaments of uniform quasi-geostrophic potential vorticity," Geophys. Astrophys. Fluid Dyn. published online (2020), 10.1080/03091929.2019.1704752.

${ }^{37} \mathrm{G} . \mathrm{K}$. Vallis, Atmospheric and oceanic fluid dynamics: fundamentals and large-scale circulation (Cambridge University Press, Cambridge, 2006).

${ }^{38}$ N. J. Zabusky, M. H. Hughues, and K. V. Roberts, "Contour dynamics for the euler equations in two dimensions," J. Comput. Phys. 30, 96-106 (1979).

${ }^{39}$ D. G. Dritschel and R. Saravanan, "Three-dimensional quasi-geostrophic contour dynamics, with an application to stratospheric vortex dynamics," Quart. J. Roy. Meteorol. Soc. 120, 1267-1297 (1994).

${ }^{40}$ D. G. Dritschel, "A general theory for two-dimensional vortex interactions," J. Fluid Mech. 293, 269-303 (1995).

${ }^{41}$ V. G. Makarov and Z. Kizner, "Stability and evolution of uniform-vorticity dipoles," J. Fluid Mech. 672, 307-325 (2011).

${ }^{42}$ J. N. Reinaud, "Three-dimensional quasi-geostrophic vortices equilibria with $m$-fold symmetry," J. Fluid Mech. 863, 32-59 (2019).

${ }^{43}$ Z. Kizner, V. Makarov, L. Kamp, and G. van Heijst, "Instabilities of the flow around a cylinder and emission of vortex dipoles," J. Fluid Mech. 730, 419-441 (2013). 